\title{
Psicología de la comprensión textual y control de la comprensión: revisión de conceptos
}

\author{
Psychology of Textual Comprehension \\ and Comprehension Monitoring
}

Geral Eduardo Matéus Ferro*

Resumen

Se presentan algunos conceptos básicos de la comprensión textual, desde la psicología del discurso, y se describe la estrategia metacognitiva control de la comprensión, como una propuesta de investigación psicológica sobre la comprensión textual. Para esto, en primer lugar, se desarrollan conceptos relacionados con la comprensión del texto, los niveles de representación textuales y el procesamiento en ciclos; enseguida, se introduce la noción de metacognición; luego se explica la estrategia metacognitiva de control de la comprensión y se analizan algunas de las variables investigadas asociadas con dicha estrategia; finalmente, y a modo de conclusión, se analiza la relación entre el control de la comprensión y el rendimiento académico.

Palabras clave:

Cognición, comprensión textual, control de la comprensión, evaluación, metacognición, regulación.

\section{Abstract}

Presented are some basic concepts of textual comprehension from the physcology of discourse. The description of a metacognitive strategy of comprehension monitoring is presented as a psychological research method for textual comprehension. In this sense the article develops some concepts relating to textual comprehension, representation levels, and processing cycles, afterwards metacognition is introduced. Then the metacognitive strategy of comprehension monitoring is explained and a set of related variables are analyzed. Finally, as a conclusion, links between comprehension monitoring and students achievement are analyzed.

\section{Key words:}

Cognition, comprehension monitoring, evaluation, metacognition, regulation, textual comprehension.

Artículo recibido el 24 de agosto de 2007 y aprobado el 31 de octubre de 2007

* Profesor del Departamento de Lenguas de la Universidad Pedagógica Nacional gmateus@pedagogica. edu.co

Estudiante de psicología del lenguaje en la Universidad de Alcalá (España), con el apoyo del Programa Alßan, Programa de Becas de Alto Nivel de la Unión Europea para América Latina, beca No E06D101249CO, y de la Universidad Pedagógica Nacional. ${ }^{*}$ 


\section{Introducción}

Desde el salto que dio la lingüística a finales del siglo XX hacia el estudio de unidades supraoracionales, se abrió la perspectiva de análisis de las producciones lingüísticas al discurso y al texto. No obstante, vale la pena recordar que con anterioridad otras disciplinas -filosofía, pragmática, sociología, etnología y antropología - ya estudiaban y analizaban unidades discursivas. Pero es de resaltar que el objeto de estudio de algunos lingüistas, a partir de este momento, se amplió hacia unidades mayores y surgieron como relevantes problemas relativos a la estructura textual y a su proceso de comprensión y producción.

En esta revisión conceptual se perfila una de las vertientes de análisis de la comprensión del discurso ${ }^{1}$ desde la psicología, la estrategia metacognitiva del control de la compresión, presentándola como una alternativa de investigación con variadas aplicaciones, especialmente relevantes en el ámbito educativo.

En este sentido se presenta el concepto de comprensión en niveles de representación y se examina la "versión" psicológica de algunos conceptos propios del paradigma textual (por ejemplo, macroestructura, microestructura, los cuales son tangencialmente comparados con sus análogos en lingüística). También se describe la estructura que subyace a los textos y su procesamiento desde una perspectiva psicológica a partir de las propuestas de Walter Kintch (1998) y Teun A. van Dijk (1983) especialmente.

Así mismo, se exponen los supuestos teóricos psicológicos relativos a la estructura textual y su procesamiento, los cuales soportan algunas líneas de investigación en la comprensión del discurso y, específicamente, explican la elección de las variables investigadas en la comprensión textual, el marco del estudio de la estrategia metacognitiva del control de la comprensión.

Al final del artículo se presenta, a modo de conclusión, una serie de interrogantes surgidos en los datos y resultados de investigaciones realizadas en

1 Dado que en la psicología no se hace diferencia entre texto y discurso como sí se hace en lingüística, se emplean indistintamente los dos términos en este artículo. los contextos español y portugués, y quizás aplicables a nuestro ámbito colombiano.

\section{Comprensión}

Comprender textos, desde una perspectiva psicológica, supone más que una tarea lingüística de decodificación de signos escritos en unidades semánticas, pues en la estructura superficial del texto no se explicitan todos los elementos necesarios para su comprensión. La tarea del lector consiste en ir más allá de los signos verbales, esto es, crear y reconstruir informaciones que llenen los "vacíos" dejados por los signos escritos, con el fin de recrear en la mente el significado del texto. En consecuencia, Walter Kintch (1988) ha propuesto un modelo que tiene en cuenta las actividades que el sujeto realiza cuando comprende un texto, la estructura que subyace al texto y la integración de estas actividades con dicha estructura.

\section{Niveles de representación}

Para explicar el proceso que lleva a la representación de un texto por parte de un sujeto, Kintch propone, entonces, un modelo de la comprensión organizado en niveles de procesamiento que permite entender cómo se comprende, recuerda y piensa con el lenguaje. Según este autor, el resultado de la comprensión es una representación proposicional compleja que se puede explicar en niveles y que refleja las relaciones semánticas establecidas en este proceso.

El texto en sí está integrado por un conjunto de elementos que se perciben directamente; este conjunto de elementos conforma el texto de superficie. Las proposiciones que se derivan directamente del texto superficial constituyen el nivel base del texto, el cual corresponde al primer nivel de representación. Adicionalmente, en la comprensión textual, los lectores añaden a esta representación del nivel base del texto, o texto base, información propia, proveniente generalmente de la memoria de largo plazo (MLP). A esta nueva representación, que integra la información explícita del texto con la aportada por el individuo, Kintch la denomina modelo de situación. Esta nueva información, aportada por el individuo, comprende tanto los recuerdos, evo- 
caciones y asociaciones "generadas" a partir de la información representada en la base del texto, así como la información construida por el individuo para llenar los vacíos del texto (inferencias 2 ). La figura 1 ilustra los niveles de representación textuales del modelo de Kintch.

Figura 1. Niveles de representación textual.

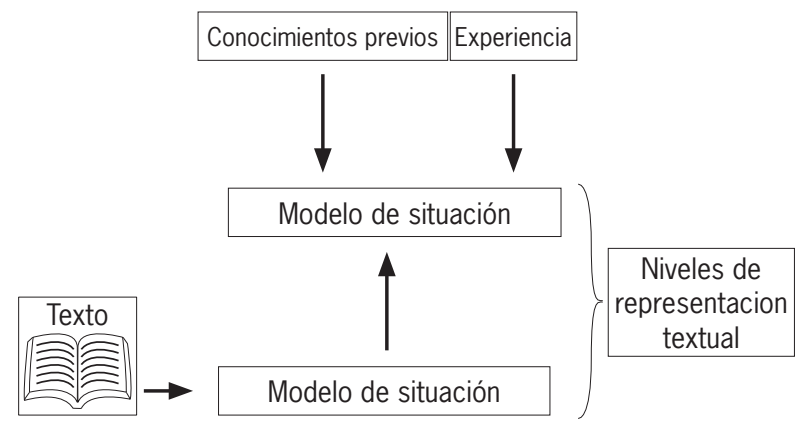

Superficie

Es de resaltar que según este modelo, todos los elementos que integran la representación del texto son formalizables en términos de proposiciones y, además, que es posible distinguir las proposiciones correspondientes en cada nivel -base del texto y modelo de situación - y sus relaciones. Las primeras se corresponden y, en algunos casos, se derivan de los elementos explícitos del texto de superficie, en tanto que las proposiciones del modelo de situación implican la creación e integración de nuevos elementos en las representaciones.

\section{La organización del texto}

Otro aspecto que es preciso tener en cuenta corresponde al hecho de que el texto también es susceptible de ser analizado en su estructura de significado. En

2 Desde la perspectiva psicológica, a diferencia de la lingüística, se consideran inferencias todas aquellas tareas de construcción de información que suponen la creación de vínculos mentales entre los elementos que se perciben mediante sistemas perceptivos, como el visual, por ejemplo, en el caso de la lectura de textos escritos. En esa medida, desde la psicología de la comprensión textual, son inferencias tanto las relaciones correferenciales, anáforicas y catafóricas, como las evocaciones de esquemas de experiencia, entre otras. No obstante, hay propuestas de clasificación que especifican diferentes tipos de inferencias; al respecto se pueden revisar los trabajos de Manuel Gutiérrez-Calvo (1999) y José Antonio León (2003). un trabajo previo, Kintch y Van Dijk (1983) propusieron dos tipos de estructura que subyacen al texto: la microestructura y la macroestructura ${ }^{3}$.

La microestructura hace referencia a la estructura de significados locales, expresados en cada oración del texto. De ésta surge una representación mental detallada del texto que permite establecer relaciones locales entre las proposiciones, fundamentadas en la atribución de referencia entre ellas, es decir, en que comparten los mismos referentes.

Por su parte, la macroestructura se puede entender como una serie jerárquica de proposiciones generales, que se desprenden de la microestructura, y constituye la estructura de significado global del texto. En resumen, ésta recoge las unidades conceptuales básicas de la microestructura y las integra en otra mayor.

En algunas ocasiones, los textos incluyen oraciones que expresan esta macroestructura, sin embargo, en otras debe ser inferida por los sujetos. Un buen resumen de un texto debería, idealmente, expresar su macroestructura.

También es preciso señalar que cualquiera de las representaciones que integran estas estructuras semánticas del texto (micro y macro) puede generar la creación o evocación de otros conocimientos por parte del sujeto, y constituirse, junto con las representaciones de la información explícita, en una estructura de comprensión más compleja: el modelo de situación. Así, no hay una correspondencia directa entre representación del nivel de base y microestructura, por un lado, y entre la representación del modelo de situación y macroestructura, por el otro, sino que los dos niveles de representación

3 En este punto también es preciso notar que el tratamiento de los dos conceptos difiere en la psicología y la lingüística. En la primera, las dos estructuras corresponden a unidades conceptuales, se analizan como entidades psicológicas y se describen las tareas implicadas en su procesamiento. Por su parte, desde la lingüística, mientras que la microestructura atañe a un nivel anterior al conceptual y comprende aspectos sintácticos y semánticos de superficie que implican grados de cohesión, la macroestructura es básicamente de carácter semántico y define la coherencia textual. Además, las características generales asociadas a la superestructura en lingüística, por ejemplo, el carácter descriptivo, narrativo o argumentativo de un texto, en la psicología del discurso se analizan como aspectos de la macroestructura. 
poseen elementos de las dos estructuras, tal como se representa en la figura 2.

Figura 2.

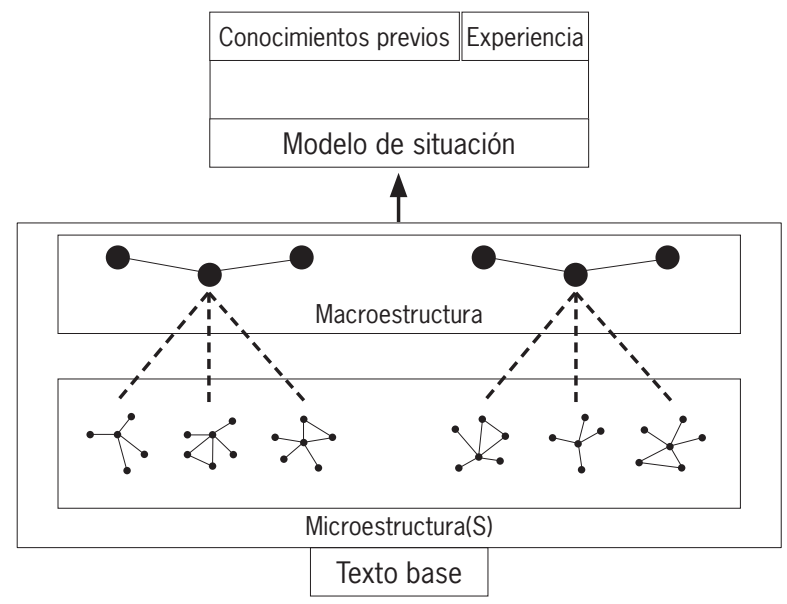

Según Kintch (1988), tanto el nivel de base como el del modelo de la situación poseen su propia micro y macroestructura, obviamente relacionadas. Aunque se postulan distintos niveles y estructuras, el individuo crea una sola representación del texto; claro está que dicha representación es compleja y analizable en los mencionados niveles y estructuras, y puede variar con el tiempo.

\section{Procesamiento en ciclos}

Además de los niveles de procesamiento y la organización propia del texto, también es posible describir el proceso mismo de comprensión. Uno de los modelos más reconocidos es el de construcción-integración (CI), también propuesto por Kintch (1988).

Según este autor, cuando se empieza la lectura, se inicia el procesamiento por ciclos. Generalmente, estos ciclos tienen correspondencia con la organización propia del texto. Así, por ejemplo, la comprensión de la primera oración constituiría el primer ciclo de procesamiento. Como resultado de esta tarea, se construye una representación proposicional de los elementos que hacen parte de la misma y se guarda en la memoria de corto plazo. Esta representación incluye tanto los elementos extraídos del texto -ideas explícitas- como las inferencias realizadas por el sujeto lector.

Enseguida, se procesa el siguiente fragmento del texto que, como ya se mencionó, puede ser la siguiente oración. Una vez se construye la nueva representación proposicional, se evoca los elementos de la representación anterior que son necesarios para la comprensión de la nueva oración y se integran en una nueva representación. De esta manera, los elementos nucleares (explícitos o inferidos), cuya representación se ha elaborado en el ciclo anterior, se conservan disponibles en la mente de los sujetos y soportan la comprensión de los siguientes ciclos.

El modelo propone, entonces, que no es necesario construir de nuevo la representación de estos elementos "repetidos", sino que los componentes ya representados en un ciclo y que son necesarios para el siguiente, simplemente se integran a la nueva representación. Igualmente, lo que el sujeto realiza mientras lee -inferencias y asociaciones-, también se integra a la representación en curso.

De esta manera, el procesamiento de textos se explica a través de ciclos continuos de construcción e integración de representaciones de información, proveniente tanto del texto como de la mente del sujeto. Este proceso, más que una simple suma de elementos, se propone como una red que se enriquece a medida que la actividad cognitiva transcurre (figura 3).

Figura 3. Simulación del proceso de procesamiento, según el modelo $\mathrm{Cl}$.

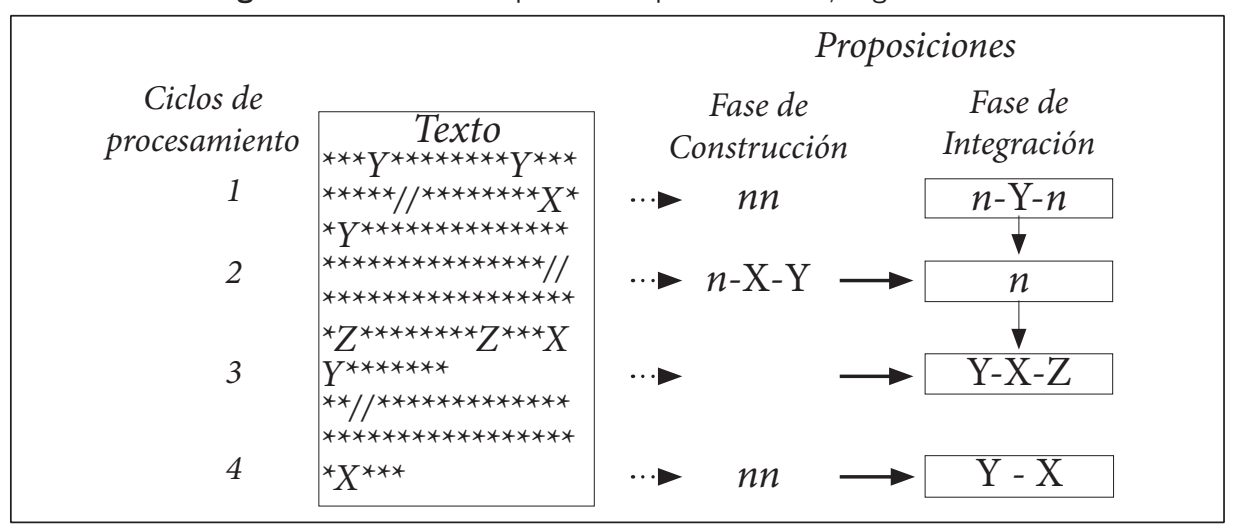


En cada ciclo de procesamiento se construyen representaciones proposicionales de las oraciones del texto (separadas por //). Los elementos más relevantes se conservan en la memoria y se van manteniendo en la mente a medida que se referencian en el texto.

Así, en esta simulación, en el primer ciclo, el elemento Y es el más informativo y se construye una representación del mismo junto con otros elementos (n) en una proposición. En el segundo ciclo, la representación de Y se evoca rápidamente al ser nombrado, y se integra a la nueva proposición construida con otro nuevo elemento X. En el tercer ciclo se introduce otro elemento Z, sin embargo al integrarse en la nueva representación están más disponibles los elementos Y y X. En el ciclo final, se conserva la representación de $\mathrm{Y}$, que se integra a la proposición, a pesar de no ser nombrado, dada su persistente aparición en los anteriores ciclos.

En general, los anteriores conceptos resumen uno de los modelos psicológicos de la comprensión más aceptado por los psicólogos del lenguaje, que recoge tanto la estructura del texto mismo como su procesamiento. En el siguiente numeral, se presenta una de las muchas posibilidades de investigación, el estudio de las preguntas, fundamentada en las anteriores propuestas de Kintch, y que considera la propuesta psicológica como válida para entender algunos de los obstáculos de los lectores en el proceso de comprensión textual.

\section{El estudio de las preguntas en la comprensión textual}

Un camino que se abre para investigar las dificultades en la comprensión, analizable en términos de la perspectiva psicológica de la comprensión, se fundamenta en el análisis de las preguntas que se suscitan en la mente de los sujetos mientras leen.

Dos investigaciones, la de Costa et ál. (2000) y la de Otero y Graesser (2001), analizan las preguntas que formulan los estudiantes al leer textos y proponen este procedimiento como herramienta para develar qué "aspecto" de lo que se lee genera dificultades en su representación. En términos generales, el procedimiento en las dos investigaciones es semejante. Después de leer textos expositivos en ciencias, se pide a los individuos que escriban las preguntas que les suscite el texto, luego se analizan y clasifican, según los niveles de representación textual al cual corresponden dichas preguntas.

Los autores hallaron que es posible diferenciar preguntas que inquieren por elementos propios de la representación en el nivel de base o en el modelo de situación, expresados en unidades distintas: palabras, oraciones y relaciones. Algunas de sus conclusiones son las siguientes:

- Es muy común que se generen preguntas por el significado de palabras, bien porque no se conoce la palabra, texto base, o porque su referente no se puede integrar a un modelo de situación.

- Las preguntas que surgen de oraciones dependen del conocimiento del lector. Por ejemplo, en algunos casos el lector no puede entender el significado de una oración porque no es capaz de crear una representación asociada a un referente a partir de lo leído -base del texto-. Distinto es el caso cuando la información de la oración discrepa con los conocimientos previos. Estas preguntas evidencian inconsistencias, sino contradicciones, entre la representación del modelo de situación, fundamentado en la experiencia del individuo y lo que propone el texto.

Otro tipo de preguntas revelan dificultades para relacionar las informaciones de distintas partes de los textos. Algunas se generan en la incomprensión de enlaces explícitos entre las proposiciones del texto o en la incapacidad para integrar las proposiciones, de manera funcional, al modelo de la situación subyacente al texto.

Hasta aquí se ha hecho un sumario de algunos conceptos fundamentales para entender la perspectiva psicológica de la comprensión de textos y un ejemplo práctico de su aplicación. Enseguida se presenta otro aspecto fundamental de la cognición -la metacognición-, que se describe primero de manera general y, luego, específicamente, a través de su relación con la comprensión de textos a través de la estrategia "control de la comprensión". 


\section{Metacognición}

Un concepto fundamental para entender el funcionamiento de los procesos cognitivos se debe a John Flavell (1979), al resaltar el estudio del conocimiento que el sujeto tiene de aquello que sabe, es decir, del conocimiento metacognitivo.

Según Hacker (1998, p. 3), lo fundamental del concepto de metacognición es la noción de "pensar sobre los propios pensamientos": pensar sobre lo que uno sabe (conocimiento metacognitivo), sobre lo que se hace en el momento (habilidad metacognitiva), o sobre un estado afectivo o cognitivo (experiencia metacognitiva). Para los autores, el valor de este conocimiento lo constituye el hecho de que "surge del interior del individuo".

De acuerdo con el modelo planteado por Flavell, la habilidad para controlar una amplia gama de tareas cognitivas se produce a través de las acciones e interacciones entre cuatro clases de fenómenos:
a. El conocimiento metacognitivo.
b. Las experiencias metacognitivas.
c. Las metas (o tareas).
d. Las acciones (o estrategias).

A su vez, el conocimiento sobre estos cuatro fenómenos se fundamenta en:

- el conocimiento, incluso en las creencias, acerca de la naturaleza y capacidades propias, o de otros, para realizar tareas cognitivas;

- el reconocimiento de la tarea que se requiere realizar y de lo que se necesita para llevarla a cabo;

- el conocimiento de las estrategias necesarias para cumplir los objetivos y de la serie de acciones (estrategias) para desarrollarla;

- la capacidad para revisar el proceso mismo, su progreso y dificultades.

En consecuencia, la metacognición incluye tanto el monitoreo activo (evaluación), como las consecuentes regulación y planeación de procesos cognitivos para alcanzar las metas. Estas actividades pueden, luego, convertirse en automáticas e inconscientes (Hacker, 1998, pp. 5 y 7).

\section{Procesos ejecutivos}

Con base en los planteamientos de Flavell, Kluwe (1982, citado en Hacker, 1998) planteó que las actividades llamadas metacognitivas comprendían conocimientos declarativos y procedimentales. Así, afirma que los pensamientos y saberes sobre lo que uno mismo, u otros saben, constituye el conocimiento declarativo metacognitivo, en tanto que aquellos pensamientos en los que se revisa y regula el curso del propio pensamiento equivale al saber procedimental (Hacker, 1998, p. 8). Específicamente, la estrategia de control de la compresión (CC) se soporta en lo que Kluwe denomina conocimientos procedimentales o procesos ejecutivos; estos implican la evaluación y la regulación de los procesos de pensamiento.

Los procesos de evaluación son aquellos "dirigidos a la adquisición de información acerca de los procesos de pensamiento de las personas". Incluyen decisiones que ayudan a: a) identificar la tarea que se realiza en el momento; b) revisar el progreso actual de dicha tarea, y c) predecir el resultado posible, según dicho progreso.

Mientras tanto, los procesos de regulación son "aquellos dirigidos a orientar el rumbo del propio pensamiento". Incluyen decisiones para: a) asignar sus recursos a la tarea actual; b) determinar el orden de los pasos que se deben seguir para completar la tarea, y c) fijar la intensidad o d) velocidad con la cual debe realizarse la tarea (Hacker, 1998, p. 9). (figura 4).

Figura 4. Procesos metacognitivos ejecutivos implicados en la evaluación y regulación.

\begin{tabular}{|c|c|c|c|}
\hline & Evaluación & $\begin{array}{l}\longrightarrow \mathrm{a} . \\
\longrightarrow \mathrm{b} . \\
\longrightarrow \mathrm{c} .\end{array}$ & $\begin{array}{l}\text { Identificar la tarea } \\
\text { Seguir el progreso } \\
\text { Predecir resultados }\end{array}$ \\
\hline $\begin{array}{c}\text { metacognitivos } \\
\text { ejecutivos }\end{array}$ & Regulación & $\begin{array}{l}\longrightarrow \mathrm{a} . \\
\longrightarrow \mathrm{b} . \\
\longrightarrow \mathrm{c} . \\
\longrightarrow \mathrm{d} .\end{array}$ & $\begin{array}{l}\text { Asignar recursos } \\
\text { Determinar el orden } \\
\text { Fijar la intensidad, o } \\
\text { fijar la velocidad con la } \\
\text { cual se realiza la tarea }\end{array}$ \\
\hline
\end{tabular}

En cualquier caso, Hacker concluye:

[...] parece haber el consenso general de que una definición de metacognición debería incluir, por lo menos, estas nociones: conocimiento sobre el propio conocimiento, los procesos y estados cognitivos y afectivos; y la habilidad para monitorear 
y regular los propios conocimientos, procesos y estados cognitivos y afectivos, de forma consciente e intencionada (Hacker, 1998, p. 11).

Así, con fundamento en la especificidad de las habilidades y conocimientos metacognitivos, en el siguiente apartado se describe la estrategia de control de la comprensión, dando cuenta de las dos fases que la integran -evaluación y regulación-; éstas se ilustran en el proceso mismo de la comprensión lectora, a partir de evidencias empíricas.

\section{La estrategia de control de la comprensión}

Consecuentemente, esta estrategia pretende estudiar la comprensión en dos fases: evaluación y regulación, dando cuenta, por ejemplo, de si el sujeto comprende o no lo que lee, los errores que comete durante la lectura, si los descubre o no, y de las tareas que realiza para solucionar los fallos cuando éstos se detectan.

\section{Evaluación de la comprensión}

Como ya se mencionó, la primera fase del control de la comprensión es la evaluación de la misma. Esta consiste en la detección o no de problemas o fallos en la comprensión. La detección de inconsistencias se ha investigado de varias maneras: a través de reportes verbales después de la lectura; solicitando a los lectores que subrayen los problemas encontrados en el texto; por medio del registro de relecturas y tiempos de lectura en los fragmentos que se suponen problemáticos. La mayoría de los estudios ha demostrado que tanto jóvenes como adultos tienen dificultades para hallar inconsistencias en la lectura (Otero, 1998).

Una posible explicación de una evaluación poco adecuada puede ser, por ejemplo, que los lectores no integran la información de las proposiciones individuales con el tema general del texto. Otras razones explicativas de estos fallos en la evaluación residen en la incomprensión de elementos léxicos y gramaticales, o en la incongruencia entre el conocimiento previo y el expresado en el texto (cfr. "El estudio de las preguntas en la comprensión textual”).

En la investigación de Otero y Campanario (1990) se estudió la evaluación utilizando textos científicos que incluían contradicciones explícitas. Después de leer, los estudiantes debían reportar si habían tenido problemas en la comprensión, subrayando la frase o frases de difícil comprensión. El 49\% de los estudiantes manifestaron no haber hallado las contradicciones. Hecho que indica una deficiencia en la evaluación de la comprensión. Una explicación plausible de estas lecturas que pasan por alto contradicciones radica en la creación de una representación no adecuada para lo leído, fundada en un procesamiento secuencial de la información. Un sujeto que lee e integra la información con sus propios conocimientos, tiene disponible en su mente lo que ha procesado más fácilmente que aquel que simplemente suma informaciones a las demás. En este último caso, la memoria de trabajo sobrepasa su capacidad y no se advierten las relaciones entre la información y, mucho menos, si hay o no contradicciones, por tanto, se evalúa mal el proceso de comprensión.

Solamente, cuando en la fase de evaluación se detectan problemas, se activa la fase de regulación. No obstante, el simple hecho de evaluar no implica que las soluciones que se tomen sean las adecuadas. El siguiente apartado se ocupa de estas situaciones.

\section{Regulación de la comprensión}

Como ya se mencionó, esta fase tiene lugar después de que el sujeto detecta algún problema en la comprensión. La regulación se refiere a la toma de decisiones para solucionar dicho problema. Por ejemplo, es muy frecuente que los sujetos, al encontrarse con problemas en la comprensión, relean los fragmentos o busquen información adicional para suplir el fallo. No obstante, en esta fase, en la toma de decisiones, también puede haber problemas de comprensión.

Datos interesantes que evidencian otras explicaciones para los fallos en la comprensión, particularmente en textos expositivos científicos, se aportan en las investigaciones de Otero y Kintch (1992) y Otero (2002). En éstas se comprueba la existencia de sujetos que, a pesar de detectar las contradicciones y evaluar adecuadamente, "reparan" las inconsistencias de significado elaborando inferencias incorrectas o dando un gran peso a las macroideas. Los dos procedimientos hacen que se 
genere una especie de "supercoherencia" que suple las contradicciones con información adicional o modificando el significado de lo leído. Es decir que se produce un error en la regulación.

En consecuencia, regular de manera adecuada supone que la nueva información hallada, o creada, para suplir los fallos en la comprensión sea coherente con las proposiciones derivadas del texto.

En un trabajo ya mencionado, Otero y Campanario (1990), empleando el "paradigma de contradicción"4, elaboraron un modelo de análisis en el que establecieron tres categorías que describen el comportamiento de los estudiantes en la lectura de textos con contradicciones explícitas, en las fases metacognitivas de evaluación y regulación:

- La primera categoría agrupa aquellos estudiantes ausentes de evaluación. Aquí los estudiantes bien creían haber entendido todo, por tanto no detectaban las contradicciones, o bien sentían que el texto era incomprensible, sin indicar explícitamente las contradicciones, y no presentaban interés alguno por resolver esta "sensación" de incomprensión.

- En la segunda categoría, los autores incluyen a los estudiantes que realizaban una adecuada evaluación pero no se regulaban, y otros que a pesar de evaluar de manera apropiada su proceso de comprensión, es decir, de hallar las contradicciones, realizaban una regulación inadecuada, por ejemplo, ante afirmaciones evidentemente contradictorias acudían a ideas tales como "la ciencia ha avanzado tanto que algo que antes no era posible ahora sí lo es".

- El tercer grupo corresponde a los estudiantes que detectaban efectivamente las contradicciones y tomaban medidas adecuadas frente a ellas, por ejemplo, calificando los textos de incomprensibles y rechazando los fragmentos contradictorios y señalándolos de manera explícita.

4 En este paradigma experimental, se introducen contradicciones explícitas en textos, en este caso científicos, sin advertir a los lectores. Después de la lectura de textos con contradicciones y sin las mismas, se les pide a los lectores que evalúen su comprensión, se les pregunta si hallaron pasajes "problemáticos" en los textos, si detectaron las contradicciones y, en el caso de identificarlas, cómo solucionaron las inconsistencias, si lo hicieron.

\section{Variables que influyen en el control de la comprensión de textos}

A través de los anteriores apartados y los ejemplos presentados, se ha visto especialmente estudios en los que para analizar el control de la comprensión se introducen características específicas, por ejemplo, contradicciones en los textos. En otras palabras, estudios en los que la variable que se controla es el texto en sí. Sin embargo, se han considerado otras variables para dar cuenta del proceso de comprensión, como las asociadas a las características de los individuos y a las tareas -respecto a éstas últimas, bien sean las sugeridas para que realicen los sujetos o las creencias que éstos tienen acerca de las mismas-.

Así, por ejemplo, entre el conjunto de variables que se han investigado relacionadas con el sujeto lector están: sus concepciones de aprendizaje, los conocimientos previos, la habilidad de autopercepción, la motivación, la aptitud verbal y la capacidad para crear inferencias.

En cuanto a las tareas, se ha comprobado que el propósito de la lectura define también las características del control de la comprensión: es distinto leer con la instrucción de hallar errores o resolver problemas, que leer sin ninguna consigna. Las ideas que el sujeto tiene con respecto a la tarea hacen que lleve a cabo determinadas operaciones, en consecuencia, el proceso se modifica.

No obstante estos tres tipos de variables estudiadas, también hay factores contextuales como la materia (dominio de conocimiento) y la autoridad epistémica (a quien se atribuye la autoría de los textos), que definen la manera como los sujetos comprenden.

En este sentido, García-Arista, Campanario y Otero (1996) examinaron la influencia del tema en la evaluación y la regulación de textos. Para ello, a un grupo de estudiantes españoles se les hizo entrega de pasajes tomados de textos de la clase de ciencias y otros relativos a la clase de lengua, pero provenientes de diarios. Los dos tipos de textos incluían contradicciones explícitas. Se instruyó a los estudiantes para evaluar la comprensibilidad de los textos y anotar los problemas que encontrasen en ellos. 
Los resultados mostraron un mejor rendimiento en la evaluación de los textos de ciencias que en los correspondientes a la clase de lengua: hallaron más contradicciones y regularon mejor su comprensión en los primeros que en los segundos. Lo que indica que esta variable contextual, el tema, influyó en el proceso de control de la comprensión.

En otra investigación, Cuerva y Otero (1996) estudiaron otra variable contextual en la comprensión: la "autoridad epistémica" de los textos. Para esto, a distintos grupos de estudiantes se les entregó textos de ciencias idénticos y con contradicciones, planteando para un caso que provenían de un texto de bachillerato y, en el otro, que habían sido extraídos de textos de primaria.

Se midió el grado de detección de las contradicciones y aunque no hubo resultados significativos en cuanto a la autoridad epistémica, sí se evidenció cierta interacción de esta variable con la de género. Los estudiantes hallaron más contradicciones en los textos provenientes de una autoridad epistémica mayor, bachillerato, que en los de la autoridad menor, primaria; mientras tanto, lo opuesto sucedió con las estudiantes. Al parecer, estas últimas tienden a descender en sus estrategias metacognitivas cuando leen textos de ciencias. En cualquier caso, el efecto de la variable contextual tuvo lugar en conjunto con una variable asociada a los individuos.

Con este sumario de las variables que se han estudiado con más frecuencia en relación con la estrategia de control de la comprensión, se cierra esta revisión. Se resalta que esta estrategia se presenta como una alternativa de investigación de la comprensión que analiza la lectura en un marco psicológico y que, además, es una opción compatible con las perspectivas lingüísticas, pues la lectura es un hecho humano complejo que integra tareas específicamente lingüísticas como actividades cognitivas.

Para terminar, se presenta un apartado que recoge datos de dos investigaciones en el marco del control de la comprensión y su relación con el rendimiento académico, y que puede leerse como una reflexión final.

\section{Control y rendimiento académico}

Inevitablemente, las investigaciones en el campo del control de la comprensión y de la metacognición sugieren que estas habilidades son determinantes en la comprensión, no sólo de textos expositivos científicos, sino de textos en general y, por tanto, de las capacidades individuales. Según esto, se plantea como hipótesis que si se promueve el desarrollo de estas estrategias, se aumentaría el rendimiento académico.

En este marco, el de la relación del control de la comprensión y el rendimiento académico, se han adelantado algunas investigaciones, tanto con estudiantes españoles (Otero et ál., 1992) como con estudiantes españoles y portugueses (García-Arista et ál., 2006).

Los dos estudios revelan que a medida que se avanza en el nivel escolar las exigencias no se relacionan directamente con la capacidad de control de los individuos. Se esperaba que a medida que se ascendiera en el grado escolar, el mejor rendimiento en las calificaciones estuviese relacionado con estas habilidades metacognitivas. Sin embargo, los resultados demostraron en las dos investigaciones y en ambos contextos, español y portugués, que no es así.

Por ejemplo, se da el caso tanto de sujetos con alta puntuación en el control de la comprensión (CC) y calificaciones bajas en las clases de ciencias, como de sujetos con baja puntuación en CC que poseen calificaciones altas en ciencias. Lo preocupante de estos resultados en los sistemas educativos español y portugués es que sugieren la adaptación de los sujetos a las formas evaluación que no consideran directamente las habilidades metacognitivas, en desmedro de un avance en términos cognitivos; la evaluación parece promover en estos contextos solamente tareas repetitivas en niveles textuales superficiales.

De este hecho surgen varios interrogantes, aplicables a nuestro contexto educativo:

- En la perspectiva de los estudiantes: ¿para qué ocuparse de profundizar en la comprensión si para aprobar un curso es suficiente con la memoria?

- En la perspectiva de los docentes: cuando evalúo la comprensión textual, ¿qué nivel de repre- 
sentación privilegio?, ¿en qué fase, evaluación o regulación, tienen más inconvenientes los estudiantes cuando leen?, ¿tengo en cuenta de manera controlada los procesos psicológicos implicados en la comprensión lectora? Los datos revelados entre metacognición y rendimiento académico expuestos de los contextos portugués y español, ¿serán muy distintos de los del contexto colombiano?

\section{Bibiliografía}

Costa, J.; Caldeira, M. H.; Gallástegui, J. R. y Otero, J. (2000). An analysis of question asking on scientific texts explaining natural phenomena. Journal of Research in Science Teaching, 37, 602-614.

Cuerva, J. y Otero, J. (1996). Influence of epistemic authority and knowledge activation on the detection of inconsistencies in science texts. International Seminar “Using Complex Information Systems", UCIS'96, Poitiers, Francia.

Dijk, Teun van (1983). La ciencia del texto: un enfoque interdisciplinario. Barcelona: Paidós.

Flavell, J. H. (1979). Metacognition and Cognitive monitoring: A New Area of Cognitive-developmental Inquiry. American Psychologist, 34(10), 906-911.

García-Arista, E.; Otero, J.; Caldeira, M.H.; Prata-Pina, E.; Patricio, A. y Cardoso, E. (2006). El control de la comprensión y el rendimiento académico en ciencias de los alumnos españoles y portugueses. (pp. 83-95). En: Díaz de Bustamante, J., y Jiménez Aleixandre, M.P. (eds.). Perspectivas sobre a aprendizaxe das Ciencias e das Matemáticas. Estudios en honor ao profesor Eugenio García-Rodeja Fernández. Santiago de Compostela: Servicio de Publicaciones de la Universidad de Santiago.

García-Arista, E.; Campanario, J. M. y Otero, J. (1996). Influence of subject matter setting on comprehension monitoring. European Journal of Psychology of Education, XXI, 4, 427-441.

Gutiérrez-Calvo, M. (1999). Inferencias en la comprensión del lenguaje. En De Vega et al. (1999). Psicolinüística del español. Madrid: Trotta.

Hacker, D. (1988). Definitions and Empirical Foundations. En: Hacker, D.; Dunlosky, J. y Graesser, A. (eds.) Metacognition in Educational Theory and Practice. London/Mahwah, Nueva Jersey: Lawrence Erlbaum Associates Publishers.
Kintsch,W. (1998). Comprehension: A paradigm for cognition. Cambridge: Cambridge University Press.

Kintch W. y Van Dijk, T. (1983). Strategies of discourse comprensión. New York: Academic Press.

León, J. A. (coord.) (2003). Conocimiento y discurso. Claves para inferir y comprender. Madrid: Ediciones Pirámide.

Otero, J. (2002). Noticing and fixing difficulties in understanding science texts. En: Otero, J.; León, J.A. y Graesser, A. (eds.). The Psychology of Science Text Comprehension (pp. 281-307). Mahwah, Nueva Jersey: Lawrence Erlbaum Associates.

Otero, J. (1998), Influence of Knowledge Activation and Context on Comprensión Monitoring of Science Texts. En: Hacker, D.; Dunlosky, J.y Graesser, A. (eds.) Metacognition in Educational Theory and Practice. London/Mahwah, Nueva Jersey: Lawrence Erlbaum Associates Publishers.

Otero, J. y Graesser, A. (2001). PREG: Elements of a model of question asking. Cognition and Instruction, 19, 143-175.

Otero, J. y Campanario, J. M. (1990). Comprehensión Evaluation and Regulation in Learning from Science Texts. Journal of Research in Science Teaching, 27(5), 447-460.

Otero, J. y Kintch, W. (1992). Failures to detect contradiction in a text: what reader believe vs what they read. Psychilogical science, 3, 229-235.

Otero, J.; Campanario, J. M. y Hopkins, K. (1992). The relationship between Academic Achievement and Metacognitive Comprehension Monitoring Ability of Spanish Secondary School Students. Educational and Psychological Measurement, 52, 419-430.

Vega de, M.y Cuetos, F. (1999). Psicolingüística del español. Madrid: Editorial Trotta S.A. 